\title{
UNIVERSIDAD Y PARO: REFLEXIONES CRITICAS SOBRE EL DESEMPLEO DE LICENCIADOS UNIVERSITARIOS
}

\section{Introducción}

En la actualidad resulta difícil aludir a la Universidad, ya sea española o foránea, sin tener en mente el problema del desempleo. Efectivamente, el desempleo de licenciados universitarios se ha convertido en un problema, con su dosis de convencionalismo, que se resiste a ser abordado y resuelto dentro de unos márgenes de fiabilidad y certeza. Este desempleo, que aguarda en las ventanillas de las Universidades el momento de la entrega del título que capacita profesionalmente, ha pasado a ser una amenaza que va desde lo estrictamente colectivo a lo más íntimo de la personalidad de los sujetos que lo experimentan. El proceso de interiorización de las pautas y valores sociales se hace en este punto especialmente claro $y$, a la vez, alarmante. No se trata sólo de un fracaso en el plano exclusivamente profesional. La frustración se acusa en la vertiente psicosocial, proyectada individualmente en cada uno de los afectados. Se trata, pues, de una caja de resonancia de múltiples dimensiones, un pregón recitado en multitud de esquinas, y en el que tanto la Universidad como la sociedad salen muy mal paradas. Curiosamente, no existen demasiados estudios sociológicos en torno al gravísimo problema del desempleo de los jóvenes licenciados. Es casi lógico rehuir un campo de

\section{RếlS}


estudio con tantos vericuetos y terraplenes. El mismo universo de estudio, si lo contemplamos desde la perspectiva de la demanda del empleo, es sumamente escurridizo. No están claras las cifras, algunas de las cuales tendremos ocasión de contemplar en este ensayo. Y mucho menos claro está el modo de acceder al estudio de ese universo, desperdigado, lleno de prevenciones, que responde violentamente $-\mathrm{y}$, por tanto, de una manera científicamente inútil - a preguntas directas y al que hay que abordar en sus distintas especialidades y carreras con una gran sutileza, buscando lo latente antes que lo manifiesto.

En estas páginas expondré algunos de los criterios que puedan servir para estudiar el fenómeno del desempleo de los licenciados, repasando alguna bibliografía sobresaliente y sacando a la luz algunos datos elocuentes que sirvan de indicación para captar la magnitud y evolución de este fenómeno.

\section{Crisis económica y desempleo}

El desempleo es una de las piezas claves definitorias de lo que normalmente se llama una crisis económica. Tradicionalmente, desde los mismos albores de la economía política clásica inglesa, el desempleo fue una de las piedras angulares del sistema económico y de la reflexión teórica que sobre él se hacía.

España vive en la actualidad uno de los momentos más dramáticos en lo que al desempleo se refiere y en comparación con los demás países de su área de desarrollo económico. Pero fue hace relativamente poco tiempo cuando se desencadenó la tormenta. En efecto, en el período comprendido entre 1960 y 1974, la tasa de desempleo en España no superó nunca el 2 por 100 de la población activa, una tasa definida como «no preocupante» por los economistas y políticos. El año 1973 fue el momento desencadenante de la crisis energética más importante que haya sufrido Occidente. La crisis económica subsiguiente no se hizo esperar y, tras las importantes oleadas de desempleo que sufrió Europa a lo largo de la década de los setenta, el paro alcanzaba en el año 1980, en España, el 11,6 por 100 de la población activa. La cifra seguiría ascendiendo hasta alcanzar, en abril de 1983, los 2.174 .495 españoles parados, lo que se traduce en un 16,64 por 100 de la población activa '. En términos económicos, el subsidio del desempleo experimentó un lógico incremento, que se tradujo en una mayor participación en las cifras del Producto Interior Bruto; efectivamente, en 1974, el subsidio de desempleo supuso el 0,18 por 100 del PIB, mientras que en 1981 llegó a alcanzar el 2,6 por $100^{2}$.

Los datos son ya elocuentes. Pero conviene recordar que el problema del desempleo es, entre otras muchas cosas, un indicador sumamente valioso para

' Estadistica de Empleo, INEM, abril 1983.

2 Contabilidad Nacional de España, INE. 
captar la evolución de las formas neocapitalistas o postindustriales, como se les quiera llamar. El paro no es, en absoluto, una dimensión estrictamente económica, a pesar de que tenga una traducción directa en indicadores económicos. El trasfondo en el que surge la inquietante imagen del desempleo, en términos generales, es mucho más extenso, más rico en matices. El paro es indicador de problemas políticos y sociales tanto como económicos. La vinculación entre fenómenos sociales, en sentido lato, y económicos se hace mucho más patente cuando se aborda el problema del desempleo. Es esto lo que hizo afirmar a Kenneth Arrow, recientemente, lo que sigue: en la actual «reedificación de la estructura económica (...) sí que podría hablarse de una cierta conexión entre los fenómenos sociales y los fenómenos económicos, porque los primeros tienen efectos sobre los segundos» ${ }^{3}$. En este marco tiene que ser contemplado el paro que afecta a los licenciados universitarios en general, y en especial a los de nuestro país. Aunque hay que tener bien presente una importante cuestión: el paro de licenciados es una pieza más dentro del engranaje del paro, que afecta a todos los niveles de la estructura acupacional. En términos puramente cuantitativos, los parados españoles que estaban en posesión de un título de grado superior en abril de 1983 no suponían más del 2,5 por 100 del total de desempleados ${ }^{4}$. Pero las características especiales del paro de licenciados hacen que éste sea importante en términos cualitativos. Es muy alto el coste social, político y económico que hay tras un parado que ostente un título universitario. La Universidad es una institución social que no puede ser medida exclusivamente con parámetros economicistas. Esa es la razón por la que el tema del paro universitario es $\tan$ importante.

Pero, como decíamos líneas arriba, este tipo de desempleo no se entiende sin comprender el marco general en el que aparece el desempleo, que afecta a todos los niveles de la compleja estructura ocupacional del sistema económico de Occidente. En efecto, los países industrializados han visto cómo se incrementaba la cifra de parados registrados oficialmente a lo largo de la década de los setenta de una forma alarmante. Y ello teniendo en cuenta que ese registro oficial no recoge sino una parte del desempleo real, en un alarde inquietante del juego de los números. En los cuadros 1 y 2 aparecen reflejados los datos del paro para la OCDE en los años 1980 a 1982, junto con previsiones para 1983. En el total de la OCDE son más de 28 millones de personas las que se encontraban en paro el pasado año 1982. Mientras que la tasa de desempleo para el conjunto de la OCDE estaba situada en el 8 por 100 para el mismo año, frente al 16 por 100 aproximadamente de España.

${ }^{3}$ K. ARRow; entrevista concedida a raíz del VII Congreso Mundial de Economia celebrado en Madrid en 1983; publicada en Economistas, núm. 4, octubre 1983 , p. 27.

4 Las cifras totales son: 2.174 .495 parados registrados oficialmente, y de ellos, 54.454 en posesión de un título superior (Estadistica de Empleo, INEM, abril 1983). 


\section{CUADRO 1}

Tasas de paro de algunos países de la OCDE. Cifras totales y series anuales 1980-1983

\begin{tabular}{|c|c|c|c|c|}
\hline & 1980 & 1981 & 1982 & $\begin{array}{c}\text { (Primer } \\
\text { semestre) } \\
1983\end{array}$ \\
\hline Estados Unidos de América $\ldots \ldots \ldots \ldots$ & 7,2 & 7,5 & 9,0 & 8,5 \\
\hline $\begin{array}{llllllllllll} \\
\text { Japón } & \ldots & \ldots & \ldots & \ldots & \ldots & \ldots & \ldots & \ldots & \ldots & \ldots & \ldots\end{array}$ & 2,0 & 2,2 & 2,2 & 2,0 \\
\hline República Federal de Alemania ... ... & 3,4 & 5,0 & 6,0 & 6,5 \\
\hline $\begin{array}{llllllllllll}\text { Francia } & \ldots & \ldots & \ldots & \ldots & \ldots & \ldots & \ldots & \ldots & \ldots & \ldots\end{array}$ & 6,3 & 7,5 & 8,5 & 8,5 \\
\hline 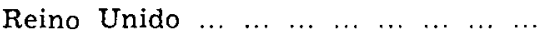 & 7,0 & 10,5 & 12,0 & 12,2 \\
\hline $\begin{array}{llllllllllll} & \operatorname{Italia} \\
\ldots & \ldots & \ldots & \ldots & \ldots & \ldots & \ldots & \ldots & \ldots & \ldots & \ldots & \ldots\end{array}$ & 7,6 & 8,2 & 9,0 & 9,0 \\
\hline $\begin{array}{cccccccccc}\text { Canadá } & \ldots & \ldots & \ldots & \ldots & \ldots & \ldots & \ldots & \ldots & \ldots \\
\end{array}$ & 7,5 & 7,5 & 8,2 & 8,2 \\
\hline 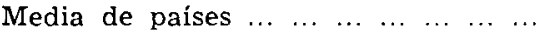 & 5,7 & 6,5 & 7,5 & 7,2 \\
\hline Otros paises de la OCDE $\ldots \ldots \ldots \ldots$ & 8,3 & 9,7 & 10,5 & 10,7 \\
\hline $\begin{array}{llllllllll}\text { OCDE-Europa } & \ldots & \ldots & \ldots & \ldots & \ldots & \ldots & \ldots & \ldots\end{array}$ & 7,0 & 8,7 & 9,7 & 10,0 \\
\hline 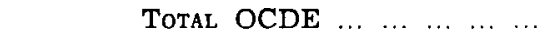 & 6,2 & 7,2 & 8,0 & 8,0 \\
\hline
\end{tabular}

Fuente: Le Defi du Chômage, París, OCDE, 1982.

\section{CUADRO 2}

El paro en la OCDE, en millones de personas. Series anuales 1980-1983

\begin{tabular}{|c|c|c|c|c|}
\hline & 1980 & 1981 & 1982 & $\begin{array}{c}\text { (Primer } \\
\text { semestre) } \\
1983\end{array}$ \\
\hline América del Norte $\ldots \ldots \ldots \ldots$ & 8,4 & 9,0 & 10,7 & 10,2 \\
\hline $\begin{array}{lllllllll}\text { OCDE-Europa } & \ldots & \ldots & \ldots & \ldots & \ldots & \ldots & \ldots & \ldots\end{array}$ & 11,4 & 14,5 & 16,0 & 16,5 \\
\hline Los cuatro grandes países europeos ... & 5,9 & 7,7 & 8,7 & 9,0 \\
\hline TOTAL OCDE $\ldots \ldots \ldots \ldots$ & 21.4 & 25,0 & 28.2 & 28,2 \\
\hline
\end{tabular}

Fuente: Le Defi du Chômage, París, OCDE, 1982.

Occidente está sufriendo reducciones de producción cada vez más acuciantes y que afectan cada vez a un mayor número de ramas industriales; los excedentes de capacidad, las reducciones de la jornada de trabajo y los despidos en masa son ingredientes casi cotidianos en el panorama económico del mundo industrializado. Las industrias químicas, sobre las que se asienta la producción de fibras artificiales, así como las industrias del textil y la confección de los países más desarrollados del Globo son un buen ejemplo 
de esa reducción de plantillas que viene siendo tónica general en el mundo industrializado. La razón parece ser ésta: la productividad de los centros industriales que tradicionalmente han venido operando en estos países ha ido perdiendo competitividad gradual e intensamente en el mercado mundial. La revolución tecnológica anunciada por el sofisticado desarrollo de la electrónica y la cibernética se ha convertido en uno de los impulsores más importantes de la pérdida de trabajo, así como de la recolocación del trabajador, con la consiguiente devaluación en su cualificación profesional. Son cada vez más importantes las inversiones que en Occidente se realizan para racionalizar la producción, con la consiguiente reducción de mano de obra, sin que se modifique la capacidad productiva. Una de las razones esgrimidas por los empresarios que siguen esa política es la de que las inversiones interiores han sufrido una importante baja en su rentabilidad, con una tendencia que no parece que vaya a modificarse en el futuro. Por otro lado, aumentan cada vez más las inversiones que los países industrializados dirigen hacia el exterior, en especial hacia los países llamados en vías de desarrollo. Se trata, fundamentalmente, de transferencias de capacidades productivas.

Curiosamente, todos los fenómenos anteriormente señalados no van acompañados necesariamente de reducciones en la producción, ni tan siquiera de reducciones de plantilla, considerando estas dos variables en un nivel puramente empresarial. En la actualidad es frecuente el caso de empresas de países industrializados que estancan o disminuyen sus inversiones, su capacidad productiva, y su número de trabajadores en el interior mientras que crecen en el exterior, especialmente en países en vías de desarrollo. El resultado de esta tendencia, en lo que se refiere a los países industrializados, es una mayor y más extraña movilidad que afecta a los trabajadores de estos países. A la búsqueda de un nuevo empleo en el mercado de trabajo nacional, los trabajadores, que carecen por lo general de la necesaria formación profesional para hacer frente al nuevo puesto $\mathrm{y}$ a las exigencias de ciertas formas productivas que requieren un alto nivel de cualificación, se encuentran sometidos a crecientes presiones para intentar mantener el valor de su fuerza de trabajo por medio de procesos de adaptación sumamente rápidos, y a veces imprevisibles, ajustados a los cambios, también a veces drásticos e imprevisibles, del mercado de trabajo.

Todo ello conduce a lo que Fröbel, Heinrichs y Kreye han dado en llamar la «nueva división internacional del trabajo» ${ }^{5}$, el marco en el que hay que considerar el fenómeno del desempleo en España, y concretamente el desempleo de licenciados universitarios.

Folker Fröbel, Jürgen Heinrichs y Otto KREYe, La nueva división internacional del trabajo. Paro estructural en los paises industrializados $e$ industrialización de los paises en desarrollo. Siglo XXI, 1980. 


\section{Universidad y desempleo universitario}

Cuando se habla de Universidad, inmediatamente acude a la mente del dialogante la imagen de la Universidad como «torre de marfil». Hace ya algunos años tuve ocasión de enfrentarme, teórica y prácticamente, con el problema que plantea el automático surgimiento de esta imagen, y creo que demostré palpablemente que, en el caso concreto de nuestra Universidad, en ningún momento pudo decirse de ella que fuese una torre de marfil ${ }^{6}$. Uno de los puntos neurálgicos del desenvolvimiento y de la justificación última de la misma existencia de la Universidad radica precisamente en eso que se ha dado en llamar la dialéctica Universidad-Sociedad. Pues bien, dicha dialéctica toma forma concreta cuando nos referimos al problema del desempleo de licenciados universitarios. La lógica puede ser planteada de la siguiente manera: teniendo en cuenta que la Universidad se abastece fundamentalmente del presupuesto del Estado (en el caso de las Universidades privadas, que se autofinancian, simplemente habría que ampliar el marco conceptual y hablar, en términos generales, de recursos sociales escasos), y dado que el capítulo de la educación es uno de los más importantes en la distribución del presupuesto, lógicamente hay que plantear la cuestión del rendimiento de la Universidad. Pero no quiero que se vea cicatería en este razonamiento; esto es, una exigencia de productividad en un sentido estrictamente material, y que poco o nada diría del carácter intrínseco de lo universitario. E1 rendimiento de la Universidad debe ser exigido en términos más amplios; en los órdenes de lo social, lo político y lo económico. Se trata, pues, de volver a plantear el tema que hace relativamente poco tiempo señalara Víctor Pérez Díaz en un excelente trabajo ${ }^{7}$, bajo la forma de debate público. Tenemos entre manos el difícil tema del obligado debate público que debe realizarse sobre una institución tan pública como lo es la Universidad. $Y$, en este caso concreto, se trata de centrar el debate sobre un asunto específico: el desempleo universitario.

Este debate, para que pueda producir el fruto que se merece, debe tomar la forma de un debate de economía política ${ }^{8}$; precisamente la forma que no adopta, por lo general. Efectivamente, el debate viene tomando normalmente dos formas distintas, aunque ciertamente paralelas: a) Un debate estrictamente económico, en el que el marco central suele ser la financiación de las Universidades. En este marco, sí cabe hablar del rendimiento más o menos productivo de la Universidad, y es aquí donde aparece con gran virulencia

- Véase mi libro La Universidad en la España de Franco, Centro de Investigaciones Sociológicas, 1981.

"Víctor PÉREZ Díaz, "Universidad y empleo", en Papeles de Economía Española, 8, 1981, pp. 296-319; véanse especialmente pp. 300 y ss.

- Puede verse un interesante desarrollo de esta idea de Economía Política en Dwayne Ward, Toward a Critical Political Economics, Goodyear Pub. Co., 1977, especialmente pp. 15-30. 
buena parte de la polémica entre las Universidades públicas y privadas, en un discurso paralelo al de la enseñanza media. b) Un debate estrictamente político, al que muy acostumbrado está nuestra Universidad. En este último, los temas principales suelen ser las relaciones de poder que gobernarán la Universidad, el control de las mismas, los intereses más o menos corporativistas que anidan en el interior de lo universitario, e incluso la pugna ideológica que se esconde tras la figura del Alma Mater.

Pero, ciertamente, no es común encontrar un enfoque de estricta y científica economía política sobre la Universidad. Y ello se echa mucho más en falta cuando se trata del problema del empleo universitario. Hemos hablado anteriormente del peso específico que los licenciados universitarios tienen en el total de las cifras registradas de paro. Ahora es el momento de profundizar algo más en aquellas primeras cifras. Con ello podremos valorar mejor la existencia real del desempleo universitario. En el cuadro 3 puede apreciarse la evolución, desde 1971, tanto del número de estudiantes universitarios como del número de licenciados que la Universidad ha arrojado anualmente al mercado de trabajo. En él se aprecian ciertas cosas que son interesantes. Para empezar, decir que, en el pasado curso 1980-81, el 1,8 de la población española estaba cursando estudios de grado superior. Se trata de una cifra que, como ha señalado recientemente Amparo Almarcha en un excelente trabajo sobre la Universidad española, «está bastante por debajo de la media, lo que implica que no podamos hablar aún de "masificación" en términos comparativos con otros países de Europa» ${ }^{9}$. Pero centrémonos en las cifras de aquellos que acaban sus estudios. Contando con las Escuelas Universitarias, acabaron sus estudios en ese mismo año escolar 76.129 personas, y si excluimos esas Escuelas, la cifra baja a 48.460 personas. Una simple suma arroja la escalofriante cifra de 138.471 personas que terminaron sus estudios superiores en los años comprendidos entre 1978 y 1981. Como puede observarse fácilmente, estos datos no concuerdan con los que hemos contemplado al hablar del paro de personas con título universitario. $\mathrm{Y}$, por lo mismo, el desfase con las cifras de los inscritos en los COIEs, que corresponden a licenciados que buscan su primer empleo, y que el lector podrá encontrar en los cuadros 8 y 9 , no puede ser más grande. Ciertamente, habrá que concluir que la inmensa mayoría de los recién licenciados no aparecen registrados en las cifras oficiales del paro registrado, constituyendo un universo desperdigado, en situaciones laborales inexistentes, ignoradas o subterráneas, lo que hace especialmente difícil un seguimiento ordenado y científico si lo que se pretende es conocer su situación. La experiencia del personal que trabaja en los COIEs podría hablar largo y tendido de este asunto. Pero eso habrá que dejarlo para el último apartado de este trabajo.

${ }^{9}$ Amparo Almarcha, Autoridad y privilegio en la Universidad española: estudio sociológico del profesorado universitario, Centro de Investigaciones Sociológicas, 1982 , p. 41. 


\section{CUADRO 3}

Alumnos matriculados en enseñanza superior y alumnos que terminaron sus estudios de cnseñanza superior. Series anuales 1971-72/1980-81

\begin{tabular}{|c|c|c|c|c|c|c|c|c|c|c|}
\hline & $1971-72$ & $1972-73$ & $1973-74$ & $1974-75$ & $1975-76$ & $1976-77$ & $1977-78$ & $1978-79$ & $1979-80$ & $1980-81$ \\
\hline 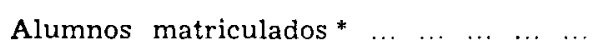 & 380.007 & 381.133 & 418.724 & 484.973 & 555.017 & 596.316 & 677.118 & 648.521 & 666.555 & 681.022 \\
\hline $\begin{array}{l}\text { Alumnos matriculados (excluyendo es- } \\
\text { cuelas universitarias) } \\
\ldots\end{array}$ & 259.017 & 284.722 & 322.043 & 366.407 & 420.648 & 445.307 & 504.912 & 477.256 & 487.725 & 501.982 \\
\hline
\end{tabular}

* Incluye escuelas universitarias, Facultades y colegios universitarios, escuelas técnicas superiores y otros estudios supe-

Fuente: Estadistica de la enseñanza, INE. 
Este volumen de licenciados plantea inquietantes preguntas. ¿Puede nuestra economía permitirse el lujo de lanzar al mercado semejante número, conduciéndoles a una situación de paro o subempleo de difícil catalogación? Planteado así, el problema rebasa con creces el ámbito del desempleo universitario y se sitúa en el terreno más amplio de la evolución de la población activa y de la política económica a seguir en los próximos años. Luis Albentosa Puche lo ha formulado recientemente, con agudeza, de la siguiente manera: «Hasta 1995 al menos, una creación neta de empleo tan reducida como la que se deriva de la implantación - aún paulatina - de la tecnología microelectrónica y un crecimiento de la población activa previsto en dos millones de personas hace ineludible que la política económica centre su atención en minimizar el paro y en paliar sus consecuencias tanto económicas como sociales» ${ }^{10}$. Enfrentamos aquí una curiosa paradoja. La tasa de parados con titulación universitaria es significativa y proporcionalmente más baja que la de aquellas personas que están en el mismo grupo generacional. Es decir, a pesar de todo, disponer de un título universitario hace que quien lo ostenta se encuentre en una posición mejor para conseguir un puesto de trabajo que la posición que ocupan sus compañeros de generación que no tienen esa titulación ${ }^{11}$. En los cuadros 4, 5 y 6 se puede contemplar alguna información útil que ilustre la relación que existe entre individuos de aproximadamente la misma generación y que enfrentan el problema del desempleo desde posiciones diferentes. En el cuadro 4 se observa la evolución del paro registrado por sexo y grupos de edad en España desde 1977 hasta 1983. El peso específico del grupo de edad, comprendido entre veinte y veinticuatro años, aproximadamente la edad en la que se está en las aulas universitarias, era del orden del 46 por 100 del total de parados en 1977 , del 21,8 por 100 en 1980 y del 24,8 por 100 en 1983 . Cifras que deberán ser corregidas, especialmente para comprender el segmento generacional de dieciocho y diecinueve años, si se quiere conseguir una correcta equiparación con la permanencia universitaria, y que dan una idea bastante clara de la difícil situación de los jóvenes menores de veinticinco años. Esta información aparece completada, especificada y actualizada en el cuadro 5. En él se ofrece la tasa de actividad y de ocupación de los distintos grupos de edad para el mes de junio de 1983. Se observa con claridad que, con respecto a la población activa, el paro que registran los grupos de edad comprendidos entre dieciséis y diecinueve años y entre los veinte y veinticuatro años es sustancialmente más elevado que el del resto de los grupos de edad. Los datos contenidos en el cuadro 6, proce-

10 Luis Albentosa Puche, El paro en España, Instituto de Estudios Económicos, 1983 , p. 260.

"Pueden encontrarse buenos análisis económico-sociales de la problemática del paro en España en términos generales, desde diferentes perspectivas, en $\mathrm{Fe}$ lipe SÁzZ FERnÁNDEZ, "Movilidad laboral y servicios públicos de empleo en España", Papeles de Economía Española, 8, 1981, pp. 270-281, y en José García López y Francisco Alvira Martín, "Los españoles y el paro", ibidem, pp. 67-85. 


\section{CUADRO 4}

Paro registrado, clasificado por sexo y grupo de elad. Medias mensuales. Series anuales 1977-1983

\begin{tabular}{|c|c|c|c|c|c|c|c|c|c|c|c|c|}
\hline \multirow[b]{2}{*}{ Grupo de edad } & \multicolumn{2}{|c|}{1977} & \multicolumn{2}{|c|}{1978} & \multicolumn{2}{|c|}{1979} & \multicolumn{2}{|c|}{1980} & \multicolumn{2}{|c|}{1981} & \multirow[b]{2}{*}{1982} & \multirow[b]{2}{*}{$1983^{*}$} \\
\hline & $\begin{array}{c}\text { Hom- } \\
\text { bres }\end{array}$ & Mujeres & $\begin{array}{c}\text { Hom- } \\
\text { bres }\end{array}$ & Mujeres & $\begin{array}{c}\text { Hom- } \\
\text { bres }\end{array}$ & Mujeres & $\begin{array}{c}\text { Hom- } \\
\text { bres }\end{array}$ & Mujeres & $\begin{array}{c}\text { Hom- } \\
\text { bres }\end{array}$ & Mujeres & & \\
\hline$\leq 19 \ldots \ldots \ldots \ldots \ldots$ & 54.914 & 39.793 & 90.386 & 70.211 & 141.869 & 115.511 & 177.639 & 149.733 & 207.608 & 174.902 & 467.587 & 521.190 \\
\hline $\begin{array}{llllllll}20 & \text { a } & 24 & \ldots & \ldots & \ldots & \ldots & \ldots\end{array}$ & 101447 & 57791 & 106.462 & 59.006 & 135.862 & 83.909 & 169.695 & 109.850 & 215.097 & 146.678 & 442.954 & 542.725 \\
\hline $\begin{array}{llllllll}25 & \text { a } & 39 & \ldots & \ldots & \ldots & \ldots & \ldots\end{array}$ & & & 193.798 & 43.933 & 226.228 & 59.038 & 274.561 & 79.224 & 332.584 & 109.927 & 517.277 & 636.967 \\
\hline 40 a 44 & 110600 & (n) & 59.508 & 10.140 & 63.242 & 12.674 & 69.418 & 15.286 & 79.758 & 18.252 & 110.199 & 119.158 \\
\hline $\begin{array}{llllllll}45 & 5 & 54 & \ldots & \ldots & \ldots & \ldots & \ldots\end{array}$ & 115.000 & 16.100 & 99.732 & 14.981 & 108.308 & 18.711 & 127.011 & 23.369 & 151.419 & 28.687 & 210.096 & 234.520 \\
\hline $\begin{array}{llllllll}55 & \text { a } & 64 & \ldots & \ldots & \ldots & \ldots & \ldots\end{array}$ & 51768 & 7173 & 58.243 & 7.915 & 60.314 & 8.957 & 68.688 & 10.364 & 85.211 & 12.937 & 116.312 & 133009 \\
\hline$\geq 65 \ldots \ldots \ldots \ldots \ldots$ & & & 3.447 & 711 & 1.947 & 625 & 1.699 & 638 & 2.248 & 900 & 2.131 & 100.000 \\
\hline Total $\ldots \ldots \ldots \ldots$ & 417.759 & 121.847 & 611.576 & 206.897 & 737.770 & 299.425 & 888.811 & 388.464 & 1.073 .925 & 492.283 & & \\
\hline TOTALES $\ldots \ldots \ldots$ & 539 & 9.606 & 818. & .473 & 1.037 & .195 & 1.27 & 7.275 & 1.56 & 3.208 & 1.872 .556 & 2.187 .566 \\
\hline
\end{tabular}

* Media anual en abril de 1983.

Funnte: Datos estadísticos, Ministerio de Trabajo, 1981, e INEM, 1982 y 1983. 


\section{CUADRO 5}

Tasas de actividad y ocupación por grupos de edad y sexo, 1983

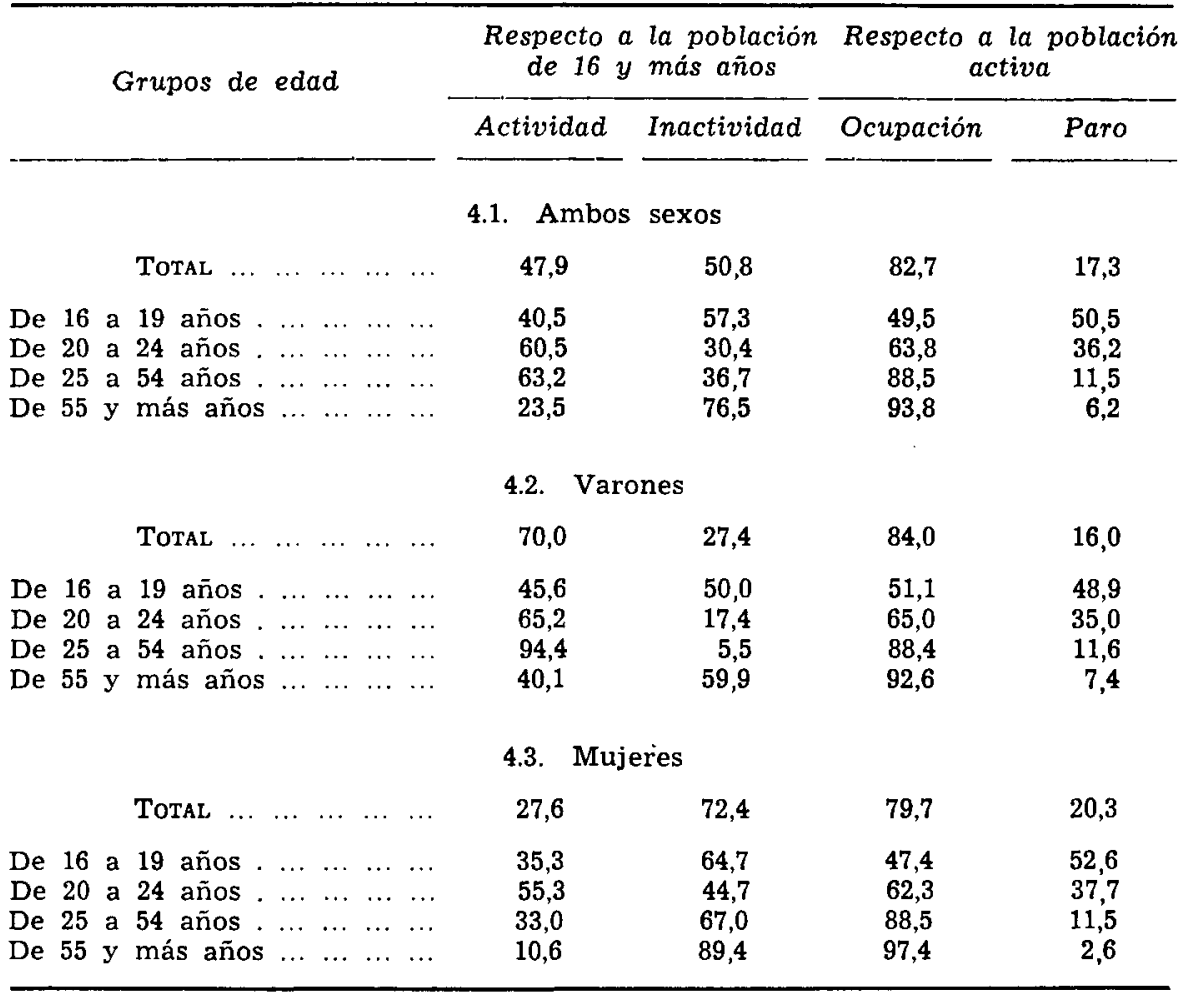

Fuente: Encuesta de Población Activa, INE, junio 1983.

dentes de una encuesta realizada por la Fundación para la Investigación Económica y Social en el año 1979, son muy elocuentes al respecto. En él se observa que la tasa de paro correspondiente a personas que responderían a lo que en este trabajo llamamos desempleo universitario arrojan una tasa de paro sustancialmente inferior a la de otros niveles educativos, fundamentalmente los correspondientes a los estudios secundarios. No obstante, esta situación parece ser peor que la que se registra en otros países industrializados. El cuadro 7 es una buena muestra de ello. Ahí se recogen las tasas de paro de los jóvenes (sin especificar edad) en una serie de países desarrollados. Como puede apreciarse, la tasa española es considerablemente más alta, seguida de cerca por la existente en Italia, Francia e Inglaterra.

Como ha señalado Víctor Pérez Díaz, hay que tener en cuenta que «el paro de titulados universitarios lo es casi exclusivamente entre titulados jó- 


\section{CUADRO 6}

Tasa de paro de la población activa española por niveles educativos, 1979

$\begin{array}{lllllllllllllll}\text { Sin estudios } & \ldots & \ldots & \ldots & \ldots & \ldots & \ldots & \ldots & \ldots & \ldots & \ldots & \ldots & \ldots & & 14\end{array}$

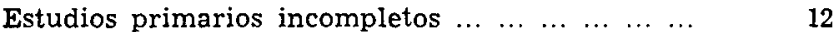

Estudios primarios completos $\left(\begin{array}{lllllll}1 & \ldots & \ldots & \ldots & \ldots & \ldots & 11\end{array}\right.$

Estudios secundarios, primer ciclo $(2) \ldots \ldots \ldots \ldots$

Estudios secundarios, segundo ciclo (3) $\ldots$...

Estudios tercer grado, primer ciclo (4) ... ....... I1

Estudios tercer grado, segundo ciclo (5) ... $\ldots \ldots \ldots$

Conjunto de la población activa $\ldots \ldots \ldots \ldots \ldots \ldots, \quad \ldots \ldots$

(1) Incluye la antigua enseñanza primaria y la actual EGB hasta $5 .^{\circ}$

(2) Incluye antiguo Bachillerato elemental y actual EGB (cursos $60^{\circ}$ y $8 .^{\circ}$ ) y primer grado de Formación Profesional.

(3) Incluye antiguo Bachillerato superior y actual BUP y segundo grado de Formación Profesional.

(4) Incluye peritajes-ingeniería técnica, profesorado mercantil, escuela de magisterio $\mathrm{y}$ otras enseñanzas que exigen para cursarse la terminación del Bachillerato superior o titulación equivalente.

(5) Incluye estudios en Facultades universitarias, escuelas técnicas superiores, estudios eclesiásticos, estudios militares y otro de igual nivel.

Fuente: Encuesta sobre el empleo, Fundación FIES, 1979 (cuadro tomado de Víctor PÉrez Díaz, "Universidad y empleo", Papeles de Economía Española, 8, 1981, p. 303).

venes», y que, por otro lado, «el problema del paro es algo menor para la población titulada (con título superior) que para la no titulada» ${ }^{12}$, tal y como se deduce de los resultados de las sucesivas Encuestas de Población Activa. No obstante, y pese a la dificultad en su realización, falta establecer criterios auténticamente científicos que correlacionen el número de licenciados que el país necesita con la situación económica en los distintos sectores. No es fácil esta cuestión. No escasean los intentos analíticos orientados en este sentido ${ }^{13}$. En el fondo, se trata de una labor de planificación que rebasa

12 Víctor PÉREz DíAz, "Universidad y empleo", Papeles de Economía Española, 8 , 1981 , p. 303.

${ }_{13}$ Podemos encontrar algunos buenos ejemplos en las siguientes referencias: Situación actual y perspectivas de empleo de doctores y licenciados en Ciencias $y$ Letras, Madrid, Ministerio de Trabajo, Dirección General de Empleo y Promoción Social, 1977; Situación actual y perspectivas de empleo de los doctores y licenciados en Ciencias Económicas y Empresariales, Madrid, Ministerio de Trabajo, Dirección General Empleo y Promoción Social, 1980; Análisis del entorno y perfil profesional del arquitecto e ingeniero español, Madrid ,ICE Politécnica, 1976; Encuesta sobre el empleo, Fundación para la Investigación Económica y Social, 1979; El empleo de los profesionales superiores, Madrid, 1976; La relación entre niveles educativos y niveles de cualificación del puesto de trabajo, Ministerio de Trabajo, 1978; Análisis causal del desempleo juvenil II: Enseñanza superior y empleo, Ma- 


\section{CUADRO 7}

Tasas de paro de jóvenes en algunos paises industrializados. Series anuales 1979-1983

\begin{tabular}{|c|c|c|c|c|c|}
\hline & 1979 & 1980 & 1981 (1) & $1982 *$ & $\begin{array}{c}\left(1 e^{e r} \text { sem. }\right) \\
1983^{*}\end{array}$ \\
\hline $\begin{array}{l}\text { Estados Unidos de Amé- } \\
\text { rica }\end{array}$ & & & $14 ?$ & & \\
\hline 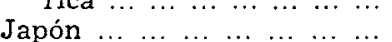 & 11,2 & 13,2 & 14,2 & 16,2 & 15,7 \\
\hline República Federal de Ale- & & $\pi, 0$ & & 7,2 & 4,0 \\
\hline $\begin{array}{lllllll} & \operatorname{mania} & \ldots & \ldots & \ldots & \ldots & \ldots\end{array}$ & 3,7 & 4,3 & 7,0 & 9,0 & 9,2 \\
\hline 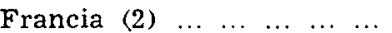 & 13,3 & 15,0 & 17,0 & 20,0 & 20,2 \\
\hline Reino Unido (3) $\ldots \ldots \ldots \ldots \ldots$ & 11,3 & 15,1 & 19,6 & 21,2 & 21,5 \\
\hline $\begin{array}{llllllll}\text { Italia } & \ldots & \ldots & \ldots & \ldots & \ldots & \ldots & \ldots\end{array}$ & 25,6 & 25,0 & 28,0 & 31,0 & 31,7 \\
\hline $\begin{array}{llllll}\text { Canadá }(4) & \ldots & \ldots & \ldots & \ldots & \ldots\end{array}$ & 13,0 & 13,2 & 13,1 & 14,0 & 14,0 \\
\hline Total países $\ldots \ldots \ldots$ & 10,8 & 12,4 & 14,0 & 15,7 & 15,2 \\
\hline
\end{tabular}

(1) Las tasas de 1981 para Francia y el Reino Unido son tasas reales. Las cifras de Japón son estimaciones basadas en tasas reales hasta el mes de octubre del año que se trate. Para el Canadá y los Estados Unidos las estimaciones están basadas en tasas reales hasta el mes de noviembre del año que se trate. Los datos de Alemania y de Italia son estimaciones.

(2) Los datos están referidos al mes de marzo de cada año.

(3) Los datos se refieren al mes de julio de cada año y comprenden a los jóvenes que dejan la escuela. La tasa de paro de los jóvenes están referidas al mes de julio, exceptuando los jóvenes que dejan la escuela; para los años 1979, 1980 y 1981 estas tasas son del 7,5, 10 y 14,8 por 100, respectivamente.

(4) Se excluyen a las Fuerzas Armadas de la población activa con edades comprendidas entre quince y veinticuatro años.

* Previsiones.

Fuente: Le Defi du Chômage, París, OCDE, 1982.

los límites de la misma Administración y se convierte en una tarea que implica a todos los sectores sociales. Está claro que, a falta de una regulación adecuada, y por muy difícil que sea de hacer, la problemática del empleo universitario recaerá, indirectamente, o bien sobre la Administración, reclutando licenciados mediante el sistema de oposiciones, a las que se abocan cientos de individuos incluso desde los últimos años de la carrera, o bien sobre el sector privado, el menos interesado, por ahora, en que exista una planificación, ya que la situación actual le permite maniobrar de una forma más relajada en un mercado de demanda de trabajo muy saturado.

Ambos sectores sociales, no hay que engañarse, están interesados tanto por la planificación cuantitativa como por la cualitativa. Efectivamente, y en

drid, Ministerio de Trabajo, 1980; Estudios diversos de SEAF-PO e INCIE sobre desempleo de licenciados; Situación actual y perspectivas de empleo de los licenciados en Ciencias Económicas y Empresariales, COIE, Universidad Autónoma de Madrid. 


\section{CUADRO 8}

Número de licenciados inscritos y numero de ofertas recibidas en los COIEs madrileños. Series anuales 1977-1982

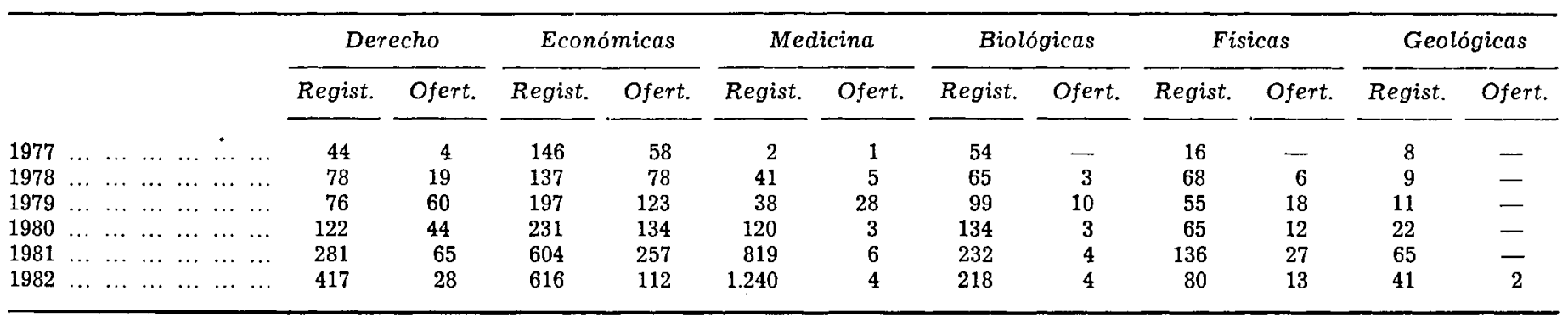

定

\section{CUADRO 8 (Continuación)}

Número de licenciados inscritos y número de ofertas recibidas en los COIEs madrileños. Series anuales 1977-1982

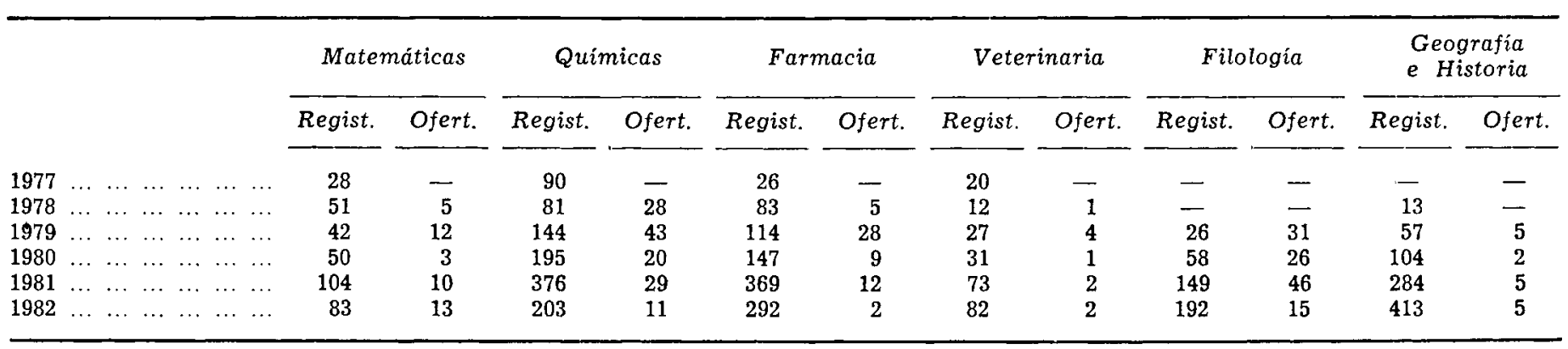




\section{CUADRO 8 (Continuación)}

Número de licenciados inscritos y número de ofertas recibidas en los COIEs madrileños. Series anuales 1977-1982

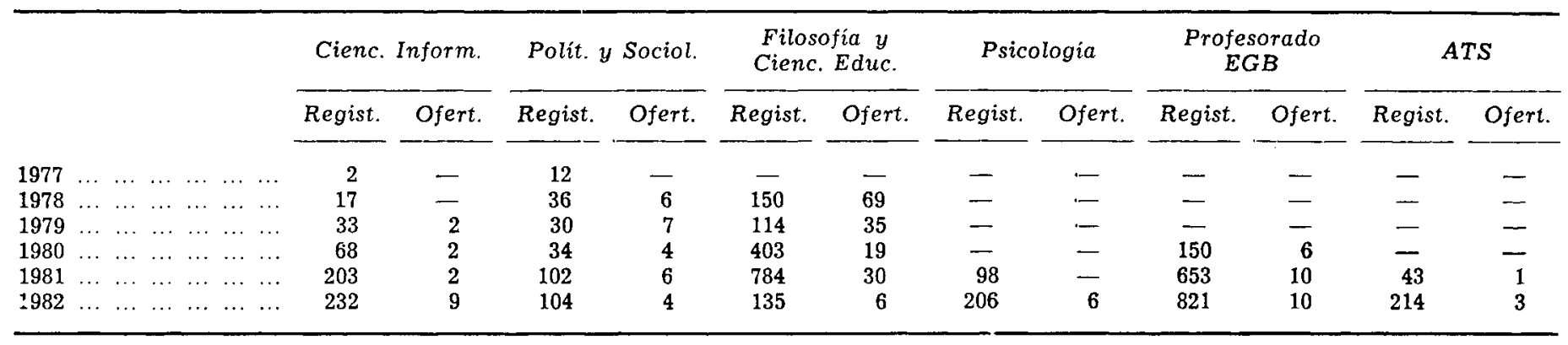

ऽ

CUADRO 8 (Continuación)

Número de licenciados inscritos y número de ofertas recibidas en los COIEs madrileños. Series anuales 1977-1982

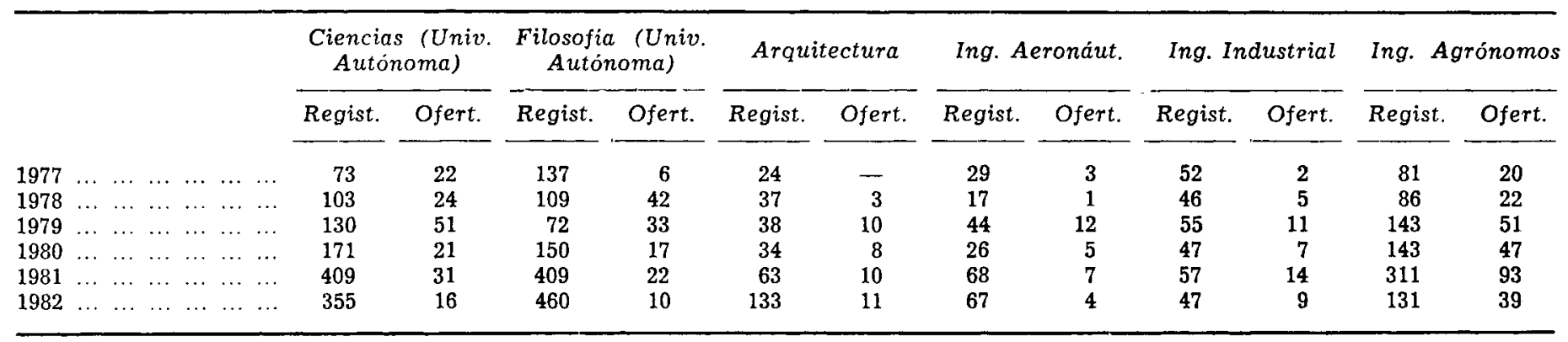


CUADRO 8 (Continuación)

Número de licenciados inscritos y número de ofertas recibidas en los COIEs madrileños. Series anuales 1977-1982

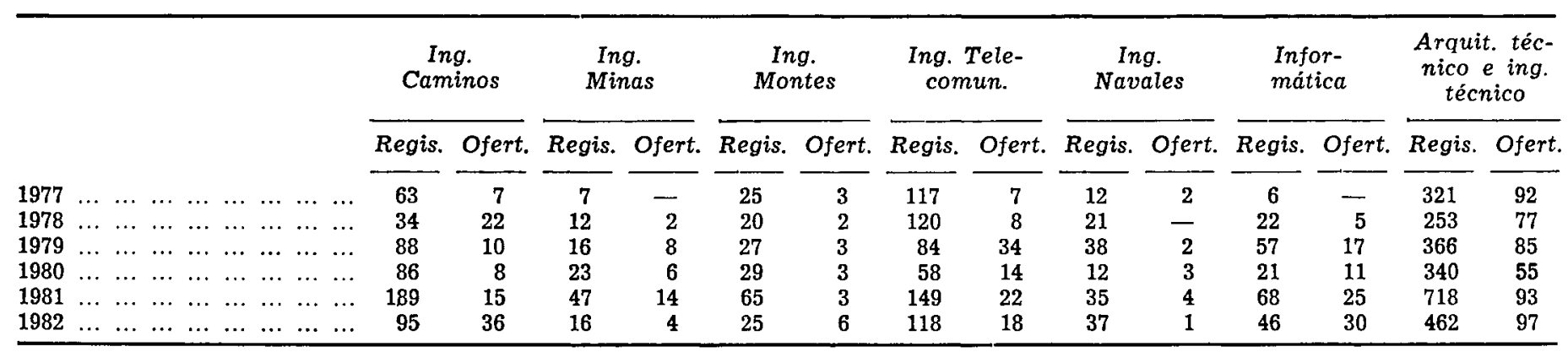

Fuente: Los COIEs de las Universidades madrileñas: seis años de actividades, Fundación Universidad-Empresa, 1983.

\section{CUADRO 9}

Numero de inscritos $y$ de ofertas de trabajo recibidas en los COIEs de las Universidades Complutense, Autónoma y Politécnica de Madrid. Series anuales 1977-1982

\begin{tabular}{|c|c|c|c|c|c|c|c|c|c|c|c|c|}
\hline & \multicolumn{2}{|c|}{1977} & \multicolumn{2}{|c|}{1978} & \multicolumn{2}{|c|}{1979} & \multicolumn{2}{|c|}{1980} & \multicolumn{2}{|c|}{1981} & \multicolumn{2}{|c|}{1982} \\
\hline $\begin{array}{l}\text { Universidad Complu- } \\
\text { tense de Madrid ... }\end{array}$ & 443 & 7 & 746 & 221 & 955 & 393 & 1.657 & 214 & 4.703 & 374 & 4.924 & 188 \\
\hline $\begin{array}{c}\text { ma de Madrid ...... } \\
\text { Universidad Politécni- }\end{array}$ & 327 & 89 & 332 & 182 & 312 & 219 & 565 & 111 & 1.507 & 195 & 1.545 & 82 \\
\hline
\end{tabular}

Fuente: Los COIEs de las Universidades madrileñas: seis años de actividades, Fundación Universidad-Empresa, 1983. 
lo tocante a la Universidad, el segundo tipo de planificación es tan importante como el primero. Una de las grandes incógnitas contenidas en el desempleo universitario es precisamente la de la formación que el titulado recibe en las aulas universitarias. La opinión más extendida, y procedente de múltiples fuentes, acusa de ineficacia a la Universidad. Los oferentes de empleo tampoco se muestran excesivamente benevolentes a la hora de elaborar un juicio tocante a esa formación profesional del recién licenciado. Por mi parte, tengo la impresión de que nos encontramos, en este punto, ante uno de esos «agujeros negros» que existen en nuestra Universidad, para el que existen más opiniones fundadas en casuísticas próximas que conclusiones razonadas procedentes del análisis de datos obtenidos mediante una metodología científica social apropiada. No abundan los estudios sobre este tema. Y, por otro lado, no es de extrañar, dadas las dificultades que entraña su abordaje. En algunos de los ya citados, la perspectiva resultante es mayoritariamente pesimista: la Universidad no prepara a sus licenciados adecuadamente para el ejercicio profesional que les aguarda cuando terminan sus estudios ${ }^{14}$. En un reciente estudio, llevado a cabo por Javier Alonso Rivas, sobre los licenciados en Ciencias Económicas de la Universidad Autónoma de Madrid parece confirmarse la misma opinión, aunque esta vez con un aire más optimista.

No hay lugar aquí para hacer un comentario profundo sobre este punto. Sí me gustaría reseñar, no obstante, algunas cuestiones sobre el mismo. Aludí antes al problema de la formación de nuestros universitarios, llamándolo «agujero negro». Me refería con esa expresión a que éste es uno de los múltiples aspectos de nuestra Universidad que, siendo importantes, resultan muy difíciles de conocer científicamente. La técnica de la encuesta no parece ser un instrumento adecuado para saber lo que ocurre; son demasiados los factores exógenos que influyen en la determinación del problema. En lo que se refiere al universo de licenciados jóvenes, la mayoría en el paro o en el subempleo, la opinión que ellos puedan tener oscila entre el desconocimiento del mercado de trabajo y toda una serie de actitudes latentes frente a lo que aprendieron en la Universidad. Llegar a ellos mediante un análisis cuantitativo parece llamar más a engaño que otra cosa. Por otro lado, está la opinión de los oferentes de empleo, sobre todo en los aspectos psicosociales de la cuestión. Esta es una dimensión que todavía está por desarrollar, y que también implicaría serias dificultades derivadas de la dispersión del universo.

El paso por la Universidad da a sus licenciados algo tan etéreo, pero a la vez tan importante, como son unas «capacidades genéricas», en atinada voz de Pérez Díaz. La experiencia de los responsables de los COIEs ratifica con creces esta idea. Pero, de hecho, sigue en pie el desafío de lograr unos datos

it Pueden verse al respecto las conclusiones que obtiene Víctor PÉrez Díaz, "Universidad y empleo", loc. cit., pp. 311 y ss. 
en este aspecto concreto que puedan ilustrar por igual a los responsables de la política educativa de la Administración y a todas aquellas personas que asumen la enorme responsabilidad de la elaboración de los planes de estudio universitarios.

\section{Los Centros de Orientación e Información de Empleo (COIE): un primer paso para administrar soluciones}

Hablar de soluciones para el gran problema que traemos entre manos resulta casi inaudito. Hay que realizar un gran esfuerzo de imaginación para ver a alguna institución resolviendo este problema, e incluso para asistir a la coadyuvación de fuerzas sociales interesadas en el tema y empeñadas en solventarlo. Cierto es que el problema del desempleo de licenciados universitarios se encuentra situado en un marco de macromagnitudes y que escapa, con mucho, a una captación global, inmediata y eficaz del mismo. Pero, por lo mismo, también es cierto que el problema es tan próximo y tan sangrante en la forma como aparece en la cotidiana vida social que se hace preciso hablar de soluciones concretas, aunque se trate de soluciones de corto vuelo, modestas en su formulación y llenas de problemas, a su vez.

Creo que es en este marco en el que tenemos que situar la figura de los Centros de Orientación e Información de Empleo (COIE), unas oficinas que son bastante desconocidas para los mismos interesados: los estudiantes de últimos años de la carrera y aquellos que acaban de terminarlas. En términos estrictamente históricos, el surgimiento de los COIEs puede ser narrado de la siguiente forma.

Los COIEs nacieron amparados en la iniciativa de la Fundación Universidad-Empresa, una institución creada en diciembre de 1973 por la Cámara Oficial de Comercio e Industria de Madrid, y por cuatro Universidades: Complutense, Autónoma y Politécnica de Madrid, y la Universidad Nacional de Educación a Distancia. Después de la realización de los consabidos estudios previos, tanto a nivel nacional como, en términos comparativos, a nivel internacional, tomando la experiencia que en este terreno tienen los Estados Unidos, se inauguró, en el primer trimestre del curso 1975-76, el COIE de la Universidad Politécnica de Madrid, al que siguieron el de la Autónoma de Madrid, en el segundo trimestre del mismo año, y el de la Universidad Complutense, en el tercer trimestre. Hay que esperar, no obstante, hasta el mes de marzo de 1977 para ver inaugurados los locales en los que desarrollarían sus funciones estos COIEs. Pero faltaba la coordinación con la Administración, especialmente con los organismos que ésta utiliza para abordar el problema del paro. Así, el 15 de julio de 1977 se realizó el acto de la firma del convenio entre el SEAF-PPO (lo que después se convertiría en el Instituto 
Nacional de Empleo, INEM, del Ministerio de Trabajo) y las Universidades Autónoma, Complutense y Politécnica, todas ellas de Madrid. En virtud de ese convenio, los COIEs se convertirian en oficinas delegadas del INEM para el tratamiento del primer empleo universitario. Aquellos convenios, vigentes durante dos años y prorrogados por un período de otros dos, regulaban la colaboración entre la Universidad y el Ministerio de Trabajo para tratar el problema del desempleo de licenciados universitarios. La idea, pues, había tomado forma. Y, al menos sobre el papel, esta forma no estaba demasiado mal construida. Una cosa importante había ocurrido en las Universidades que firmaron el convenio: a los Campus universitarios llegaba la preocupación por el desempleo de aquellos que egresaban de esos mismos Campus, y llegaba no como una volátil idea que pasa de cabeza en cabeza y de boca en boca por los pasillos y despachos de la comunidad universitaria; llegaba bajo la forma de una oficina con personal adscrito a ella, con medios administrativos y con algunos objetivos que cumplir. Entre estos últimos podríamos citar los siguientes:

1) Formalizar las demandas de empleo de los licenciados universitarios.

2) Servir de plataforma a los oferentes de trabajo para estos licenciados, que encontraban en el COIE un lugar adonde acudir para seleccionar la persona más adecuada a sus necesidades.

3) Realizar una labor activa en la prospección y búsqueda de puestos de trabajo para los licenciados. Ello se traduciría en seminarios, mesas redondas con asistencia de jefes de personal y directivos de empresas privadas, envío masivo y ordenado de propaganda explicativa de las funciones de un COIE a aquellos centros que potencialmente pudieran ofrecer trabajo, y atender los deseos de los empleadores en lo concerniente a selección de demandantes, reuniones con ellos, visitas a la Universidad por parte de directivos de las empresas, etc.

4) Atender al recién licenciado precisamente en el momento en el que precisa de mayores cuidados: cuando acaba de terminar sus estudios, el momento en que, en pocos días, cambia formalmente de status, dejando de ser estudiante y empezando a ser profesional. Es éste un paso de gran trascendencia. Hasta el surgimiento de estos COIEs, el recién licenciado debía tomar el rumbo de la oficina de empleo más próxima, donde se inscribía como demandante de empleo, a la vez que debía agudizar el ingenio para localizar no sólo ofertas de trabajo (por ejemplo, a través de la prensa diaria), sino para atender a otras alternativas muy en línea con las expectativas que en él existían; me estoy refiriendo a la oferta de becas, cursillos, realización de estudios postgrado, etc. En este sentido, el COIE vendría a cubrir esa laguna informativa, recurriendo a la documentación necesaria para realizar esa difícil 
labor de asesoramiento individualizado ${ }^{15}$. El recién licenciado, que por propia idiosincrasia se mostraba desconcertado e ignorante de las posibles salidas profesionales que su carrera tenía, encuentra en los COIEs el lugar donde obtener esa información sin coste alguno por su parte y libre de otros compromisos y responsabilidades.

5) $Y$, por fin, el COIE se convertiría en un brazo ejecutor y de conexión del Rectorado de la Universidad donde se localiza con el entorno social, precisamente en un aspecto de los más importantes que pueda desarrollar el mundo universitario: la atención a sus licenciados, el seguimiento de los mismos, apoyándoles para enfrentar la difícil prueba de la búsqueda de empleo en un momento en que el mercado de trabajo está especialmente debilitado.

Estas son algunas de las características o funciones que vendría a cubrir un COIE. Nobles y bellas sobre el papel, pero con traducción a la realidad bajo formas a veces disonantes. Volveremos dentro de un momento a revisar estas funciones. Sigamos, por el momento, con la narración en términos históricos.

En el curso 1979-80 se crea el cuarto COIE de Madrid, en la Universidad de Alcalá de Henares, siguiendo siempre la vía de la firma del convenio al que ya hemos hecho alusión. En el siguiente curso, 1980-81, se firmarían los convenios que inaugurarían los COIEs de las Universidades de Málaga, Granada y Sevilla. En el curso 1981-82 se crearía el COIE de la Universidad de Córdoba. Y en el pasado curso $1982-83$ se firmaron los convenios que corresponderían a la creación de otros centros en las Universidades de Santiago de Compostela, Politécnica de Valencia, Murcia y Oviedo. En total, doce COIEs funcionando en España, en otras tantas Universidades, y la perspectiva deseable de la instalación de tantos COIEs como Universidades existen, al objeto de convertir la figura del COIE en un centro de obligada aparición allá donde exista una Universidad.

En estos pocos años de funcionamiento, los COIEs han realizado una labor ciertamente interesante. Y me refiero no sólo a la que se plasma en reuniones o seminarios, que muchas veces tienen más de apariencia que de contenido, sino a la cotidiana y callada labor de atención a los licenciados que requieren sus servicios. Una labor que es difícil plasmar en cifras para ofrecerlas a quien quisiere leerlas, pero que, sin embargo, tiene la enorme importancia de atención y asistencia a un colectivo que está sufriendo el impacto de los graves problemas de la desocupación. Recientemente hizo su aparición en el mercado un libro-documento sobre las actividades y fundamentos de los COIEs de las Universidades madrileñas desde su fundación

is Hay que destacar en este punto la elaboración por parte de la Fundación Universidad-Empresa, con asesoramiento de expertos en las distintas especialidades, de una serie de Monografías sobre estudios universitarios que, a razón de una por carrera existente, se ha convertido en un eficaz instrumento para la labor de asesoramiento que realizan los COIEs. 
hasta esta fecha ${ }^{16}$. En él puede encontrarse la información documental precisa para calibrar el trabajo de esos centros.

No obstante, en los cuadros 8 y 9 he recogido los datos más significativos de esta actuación; esto es, el número de personas registradas en ellos, por carreras, y desde el año 1977. En el cuadro 8 puede encontrarse una paralela relación de ofertas de trabajo recibidas, también por especialidades. Son cifras brutas, ofrecidas en toda la extensión de su valor, y que reflejan por sí mismas la sensibilidad del mercado ante las diversas carreras. De destacar es Psicología, para la que no existe casi oferta de trabajo. En la misma línea están Ciencias de la Información, Políticas y Sociología, las carreras de la especialidad de Letras, en términos generales. Y, concerniente a las de Ciencias, destacan, siempre por la escasez en su movimiento, Biológicas y Geológicas. Derecho y Económicas siguen siendo las carreras que mejor se defienden en el mercado de trabajo, y ello teniendo en cuenta que el número de inscritos de Derecho es sustancialmente inferior al que realmente se corresponde con los que terminaron esos estudios, dado que la Administración se convierte en empleador nato para estos licenciados gracias al sistema de oposiciones. Parecidas razones deben ser aplicadas a los casos de Medicina y ATS, especialidades que se defienden relativamente bien, con trabajos más o menos estables (del estilo de las suplencias, muchas veces), si los comparamos con los de otras carreras para las que la salida laboral es realmente difícil.

Los datos contenidos en el cuadro 8 , junto con los del cuadro 9, en el que aparecen los números totales de personas registradas y ofertas recibidas en tres COIEs de Madrid desde el año 1977 al 1982, deben ser considerados como meros indicadores de la actividad de los COIEs y de la situación del mercado de trabajo. Por desgracia, los COIEs no son todavía centros conocidos por todos los que están interesados en lo que en ellos se hace. $Y$ esto conecta con el gran tema pendiente en este mismo momento del futuro de los COIEs.

En la actualidad, estos centros, situados en instalaciones de las distintas Universidades, con personal mixto de la Universidad y del INEM, bajo la dirección de un profesor de la Universidad y bajo la responsabilidad última, en lo que a gestión se refiere, del rector de la misma, están financiados por el INEM. Su capacidad operativa, que empieza a ser importante, no llega ni con mucho a las cotas que debiera a la luz de los objetivos anteriormente descritos. Faltan piezas importantes en el rompecabezas de estos COIEs. La Universidad, todavía no acostumbrada a entender que en su seno exista un centro que ataca frontalmente el problema del desempleo de sus licenciados, no acaba de encajar en su organigrama la figura del COIE. Mucho se ha avanzado en los últimos meses en este sentido. Pero queda mucho más por hacer. El Ministerio de Educación no ha contemplado hasta ahora la exis-

16 Javier Alonso Rrvas y Manuel López Quero, Los COIEs de las Universidades madrileñas: seis años de actividades, Fundación Universidad-Empresa, 1983. 
tencia y utilidad de estos cenrtos, y es recientemente, a raíz de una serie de entrevistas con altos responsables del Ministerio y directores de COIEs, que se ha empezado a lanzar una cada vez más necesaria idea de tutela y poten-

ciación de estos centros, con el fin de convertirlos en agentes más capaces y mejor dotados para enfrentar el problema del desempleo de licenciados. Las espadas están en alto. El paro sigue siendo uno de los principales problemas, tanto del país como de la Universidad. Y los COIEs poseen dos rotros que deben ser reconciliados entre sí al objeto de lograr la mayor operatividad posible. Por un lado, el rostro que mira hacia el INEM: la lucha contra el paro universitario no puede ser una lucha aislada de la que se está llevando a cabo por la Administración en otros frentes. Desde esta vertiente, el COIE ha venido funcionando como una Oficina Delegada de Empleo, lo que ha supuesto a veces más inconvenientes que ventajas. Por otro lado, el rostro que mira hacia la Universidad es el que tiene que ser desarrollado con más cuidado y en todas las vertientes posibles. Aquí, el COIE es un centro de orientación y ayuda al licenciado, la pieza clave para que la Universidad conecte con el mundo socioeconómico que la rodea.

En definitiva, se ha recorrido muy poco camino y queda mucho por hacer. Los COIEs españoles, que tienen paralelismo en otros países desarrollados, como los Estados Unidos (Placement Offices), la República Federal de Alemania (SOI), Francia (Cellules d'Information et d'Orientation des Etudiants y ANUDI) y Bélgica (ONEM), tienen que ser desarrollados y apoyados con mucho mayor interés por aquellos que los crearon y apoyan actualmente, si se trata de pedirles que lleven a cabo la difícil labor que les está encomendada y que tan importante es.

\section{Resumen y conclusiones}

La problemática del empleo universitario es uno de esos temas que se resisten a ser tratados desde una perspectiva unilateral, si lo que se pretende es conseguir una explicación satisfactoria del mismo. Se trata de un problema con claras vertientes políticas, sociales y económicas, que no resiste el tratamiento unilateral de ninguna de las ciencias que tratan cada una de esas tipologías variables. El empleo de los licenciados universitarios, tal y como está apareciendo en los últimos años en Occidente en general, y en España en particular, es un prototipo de tema de economía política, en su moderna acepción, y sólo en ese marco podrá ser entendido; es decir, en un marco en el que se sopesen y contrapongan variables sociológicas, económicas y políticas, en un afán quizá más de comprender el fenómeno que de explicarlo -recurriendo a la vieja terminología de Droysen-. Ya hay buenos ejemplos de este tipo de trabajo, pero todavía queda mucha tarea por hacer; sobre todo porque el fenómeno del desempleo, un fenómeno social que está situado en 
un marco de desenvolvimiento económico como el que experimentamos en el presente, resulta sumamente escurridizo y molesto.

Conviene señalar, no obstante, algunos puntos importantes que pueden arrojar luz sobre las investigaciones y reflexiones que se han realizado hasta el momento, así como las que se hagan en el futuro:

1) En primer lugar, hay que señalar los obstáculos estrictamente metodológicos que existen para el estudio de los parados con titulación universitaria. Y ello superando el nivel estrictamente economicista, adentrándonos en resbaladizos terrenos, como pueden ser el sociológico, el psicosocial y, sobre todo, el político. En efecto, el universo que integran estos licenciados muestra serios problemas de dispersión y de acceso. Las encuestas convencionales resultan poco operativas mientras no se proceda a un seguimiento estadístico apropiado desde los años de la carrera y los inmediatamente posteriores. El COIE sería el centro idóneo para realizar esa tarea de seguimiento. Pero tal y como está diseñado actualmente, con la escasez de medios materiales y humanos con los que cuenta hoy por hoy, y falto de apoyo sustancial por parte de la Universidad y la Administración, resulta prácticamente imposible escapar a las tareas burocráticas que actualmente le están asignadas para proceder a una labor que sería de gran utilidad a la hora de adoptar decisiones de política académica (selectividad y orientación a la hora de la elección de carrera).

2) Resultado inmediato de esa disparidad en el universo es la falta de coincidencia entre los datos básicos que actualmente disponemos para su análisis (EPA, INEM, etc.).

3) El problema del desempleo universitario hunde sus raíces mucho más lejos de lo que comúnmente se cree. Desde el punto de vista estrictamente universitario, debe ser contemplado buceando en las generaciones que todavía no han llegado a la Universidad. Se pueden hacer correcciones analíticas sumamente importantes desde esa perspectiva global. Y para ello hacen falta muchos más recursos de investigación de los que actualmente se cuentan. Además, el problema del desempleo universitario impacta dañinamente en esos aspectos cualitativos que antes señalábamos y que tanta importancia tienen para la sociedad. El temor del paro no sólo afecta a los estudiantes universitarios, sino que está muy presente en las mentes de los jóvenes estudiantes de BUP y COU que pretenden optar por una titulación universitaria.

4) Hay que tener en cuenta, por último, que el problema del paro universitario tiene dos frentes: oferta y demanda. Interesa conocer tanto una como otra faceta, pues son vertientes complementarias de una misma problemática.

Parece claro que el desempleo universitario es uno de los principales obstáculos que impiden el desarrollo de la labor universitaria. De la sensibilización ante la presencia de este obstáculo dependerá buena parte del futuro de nuestra Universidad. 